\title{
THEORETICAL AND EXPERT SYSTEM APPROACH TO PHOTOIONIZATION THEORIES
}

\author{
Ivan D. Petrović, Violeta M. Petrović*, Tatjana B. Miladinović \\ Department of Physics, Faculty of Science, Kragujevac University, Republic of Serbia \\ *Corresponding author; E-mail: violeta.petrovickg@gmail.com
}

(Received April 8, 2016)

\begin{abstract}
The influence of the ponderomotive and the Stark shifts on the tunneling transition rate was observed, for non-relativistic linearly polarized laser field for alkali atoms, with three different theoretical models, the Keldysh theory, the Perelomov, Popov, Terent'ev (PPT) theory, and the Ammosov, Delone, Krainov (ADK) theory. We showed that aforementioned shifts affect the transition rate differently for different approaches. Finally, we presented a simple expert system for analysis of photoionization theories.
\end{abstract}

Keywords: ionization rate, expert system, tunneling ionization.

\section{INTRODUCTION}

Non-linear ionization of atoms and molecules in an intense laser field is a currently of interest problem in atomic physics. Theoretical explanation of ionization processes begun with Keldysh, who showed in his paper (KELDYSH, 1964) that the nature of multiphoton and tunneling ionization is essentially the same, and presented the two limiting cases of non-linear photoionization. These two mechanisms are distinguished by the value of what today is widely known as Keldysh parameter $\gamma=\omega \sqrt{2 I_{p}} / F$, where $I_{p}$ is unperturbed ionization potential for considered system, $\omega$ is laser frequency and $F$ is the field strength in $\mathrm{Wcm}^{-2}$. Atomic units $\left(m_{e}=\hbar=e=1\right)$ were used throughout this paper unless otherwise indicated. If $\gamma \gg 1$ the multiphoton ionization takes place, while the tunneling ionization prevails when $\gamma \ll 1$. After the appearance of the Keldysh work, its results were refined by Perelomov, Popov and Terent'ev in Perelomov et al. (1966). Next, Ammosov, Delone and Krainov, based on PERElomov et al. (1966), derived the ADK theory (AMMOSOV et al. 1986) for the case of the tunneling ionization for arbitrary complex atoms and atomic ions.

The intent of this paper was to compare an influence of the ponderomotive potential and the Stark shift of the initial binding state of alkali atoms on the transition rate, as well as the influence of the spatial distribution of the laser beam shape. This was accomplished by analyzing three commonly used theoretical models for field ionization, Keldysh, PPT and ADK. 


\section{THEORETICAL FRAMEWORK}

The Keldysh theory provides a commonly accepted framework for a quantitative analysis of ionization processes. With the exponential accuracy on the incident laser field, the Keldysh transition rate (KELDYSH, 1964) has the form:

$$
W_{\text {Keldish }}=\frac{\sqrt{3 \pi Z F}}{2^{7 / 4}} \times \exp \left[-\frac{2 Z^{3}}{3 F}\left(1-\frac{1}{10} \frac{\gamma^{3} z^{2}}{2 l}\right)\right] \text {, }
$$

where $\mathrm{Z}$ is the charge of the atomic residue and $F=\left(27.5 / 5.1 * 10^{9}\right) \sqrt{\ln t}$.

Perelomov, Popov and Terent'ev (PERELOMOv et al. 1966) suggested a more accurate model for calculating the photoionization rate for an atom and atomic residual ions. This model takes into account the Coulomb interaction between outgoing photoelectron and ion. The PPT theory is valid for arbitrary values of the Keldysh parameter $\gamma$. It agrees quite well with the experimental results in both ionization regimes, multiphoton and tunneling (LIN et al. 2004). Based on the PPT theory, the ionization rate in linear laser field is given (VOLKOVA et al. 2006) by the formula:

$$
W_{P F T}=4 \sqrt{\frac{3}{\pi} \frac{\sqrt{8 l_{p}^{2}}}{F}} \times \exp \left[-\frac{2}{3} \frac{\sqrt{8 r_{p}^{2}}}{F}\left(\frac{3}{2 \gamma}\right)\left(\left(1+\frac{1}{2 \gamma^{2}}\right) \operatorname{ArcSinh} \gamma-\frac{\sqrt{1+\gamma^{2}}}{2 \gamma}\right)\right] .
$$

The most often used tunneling theory is the ADK theory, which is in excellent agreement with experiments in noble gases and small molecules. The ADK theory has extended the PPT theory for the tunneling ionization rate of arbitrary complex atoms and atomic ions. Transition rate formula for linearly polarized laser field in cases of zero momentum is:

$$
W_{A D K}=\sqrt{\frac{2 n_{g f^{3} F}}{\pi Z^{\Xi}}} \frac{F}{8 \pi Z}\left(\frac{4 \mathrm{\gamma}\left(2 l_{p}\right)^{\mathrm{Z} / \mathrm{z}}}{\omega Z}\right)^{2 n_{\varepsilon f}} \times \exp \left[-\frac{2}{3} \frac{2 r_{p} Y}{\omega}\right]
$$

In the case of non-zero initial momentum of the ejected photoelectrons, the ADK formula has the following form (RISTIĆ et al. 2009):

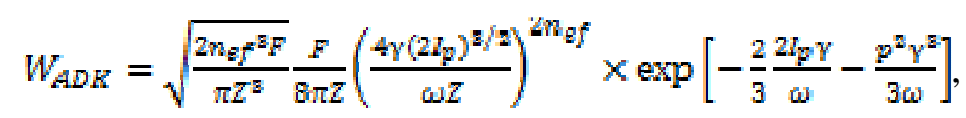

where $n_{e f}$ is the effective principal quantum number, $n_{e f f}=Z /\left(2 I_{p}\right)$ and $p$ is the initial momentum of the ejected photoelectrons (LANDAU and LIFSHITZ, 1991; BAUER, 2006).

In all of these theories the exponential dependence on field strength and the ionization potential is emphasized. However, at higher intensities the atomic level structure and the ionization process become increasingly influenced by the laser irradiation through two wellknown effects, the ponderomotive potential and the Stark shifts. In process of laser induced ionization, a bound electron must acquire energy equal to its ionization potential increased by two additional terms, one which corresponds to the ponderomotive potential $U_{p}=F^{2} / 4 \omega^{2}$ (Delone and Krainov, 1998) and second to the Stark shift $E_{s t}=\alpha F^{2} / 4$ (DELONE and KRAINOV, 2000), where $\alpha$ is the static polarizability of the atom (SCHWERDTFEGER, 2014). Accordingly, the field free ionization potential $I_{p}$ has the following form (VOLKOVA et al. 2011): $E_{\text {eff }}=I_{p}+U_{p}+E_{s t}=I_{p}+F^{2} / 4 \omega^{2}+\alpha F^{2} / 4$, where $E_{\text {eff }}$ denotes the shifted, effective ionization potential.

Putting $E_{\text {eff }}$ in Eq. 1, the Keldysh tunneling ionization rate is found to be:

$$
W_{\text {Reldish }}^{\text {eff }}=\frac{\sqrt{3 \pi Z F}}{2^{7 / 4}} \times \exp \left[-\frac{2 z^{\Sigma}}{3 F}\left(1-\frac{1}{10} \frac{\gamma^{2} z^{n}}{2\left(l_{p}+F^{2} / 4 \omega^{2}+\alpha F^{2} / 4\right)}\right)\right]
$$


where superscript eff denotes both the ponderomotive and the Stark shifts. Repeating the same procedure, $W_{p P T}^{\text {\#ff }}$ and $W_{A D K}^{\text {\#ff }}$ were obtained:

$$
\begin{aligned}
& W_{P P T}^{\theta f f}=4 \sqrt{\frac{3 \sqrt{8\left(l_{p}+F^{2} / 4 \omega^{2}+\alpha F^{2 / 4}\right)^{2}}}{F}} \times \exp \left[-\frac{2}{3} \frac{\sqrt{\left(l_{p}+F^{2} / 4 \omega^{2}+\alpha F^{2 / 4}\right)^{2}}}{F}\left(\frac{3}{2 \gamma}\right)\left(\left(1+\frac{1}{2 \gamma^{2}}\right) \operatorname{ArcSinh} \gamma-\frac{\sqrt{1+\gamma^{2}}}{2 \gamma}\right)\right]
\end{aligned}
$$

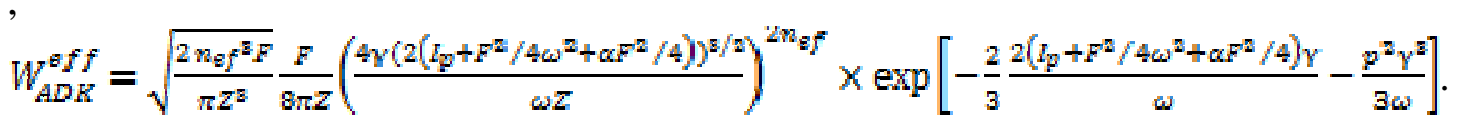

Additionally, the laser beam shape is regarded as Lorentzian (SHCHATSININ IHAR, 2009; Sheally and HoffNagle, 2006; Petrović and Miladinović, 2014):

$$
F_{L}(0)=\frac{F}{\left(1+\left(\frac{\theta}{n}\right)^{2}\right)}
$$

where $\rho$ is the axial coordinate that is normal to the direction of the light ray, $\rho=R \sqrt{1+\left(\lambda z / \pi R^{2}\right)^{2}}$ (ZHANG, 2010) and $R$ is called the laser beam waist, which represents the smallest spot size realized at $z=0$. Petrović and Miladinović (2014) have used Lorentzian and Gaussian beam shape and showed that both give satisfying results. Here we have chosen Lorentzian.

Based on Eq. 8, the effective ionization potential can be written in the following form: $E_{L, f f}=I_{p}+\left(F /\left(1+(\rho / R)^{2}\right)\right)^{2} / 4 \omega^{2}+\alpha\left(F /\left(1+(\rho / R)^{2}\right)\right)^{2} / 4$. According to Eq. 12 and the inline equation for $E_{L_{l}, \text { fff }}$, Eqs. 5, 6, and 7 can be rewritten in the following form:

$$
\begin{aligned}
& W_{\text {Keldish }}^{L, \text { eff }}=\frac{\sqrt{\ln Z(F /(1+(\rho / R) \partial)}}{2^{7 / 4}} \times \exp \left[-\frac{2 Z^{2}}{3\left(F /\left(1+(\rho / R)^{2}\right)\right)}\left(1-\frac{1}{10} \frac{\gamma^{2} Z^{2}}{2 E_{L_{i}} s f f}\right)\right] \text {, }
\end{aligned}
$$

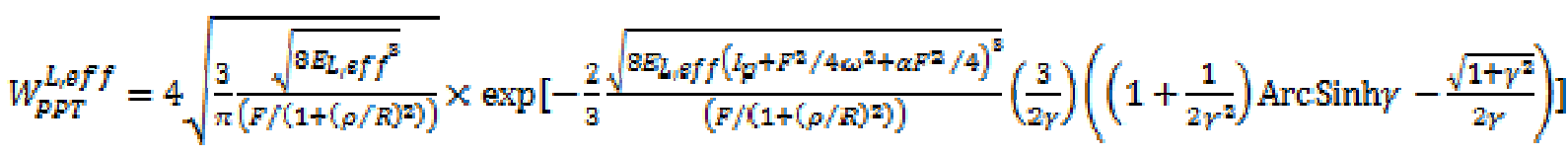

And

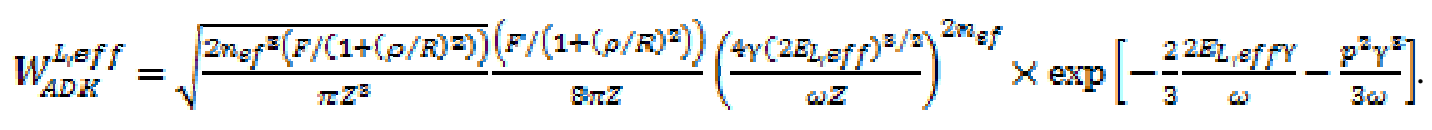

where $L$ indicates the Lorentzian distribution.

\section{RESULT AND DISCUSSION}

In this paper were theoretically analyzed the tunneling transition rates, for $\gamma=0.3$, in the field of intensity $I=10^{12}-10^{16} \mathrm{Wcm}^{-2}$, for linearly polarized Ti:sapphire laser, $\lambda=800 \mathrm{~nm},(\omega=0.05696 \mathrm{a} . u$. $)$. The single ionized, $Z=1$, alkali atom of potassium, $\mathrm{K}$ is studied. The obtained results were compared for three different ionization models. Also, the influence of spatial distribution on the transition rate is discussed.

We started from the transition rates, $W_{\text {Keldish }}, W_{P P T}$ and $W_{A D K}$ for the field-free ionization potential (see Eqs. 1, 2 and 4), for generally assumed laser beam shape, without any specifications. As a result, the following theoretical curves were obtained (see Fig. 1). 


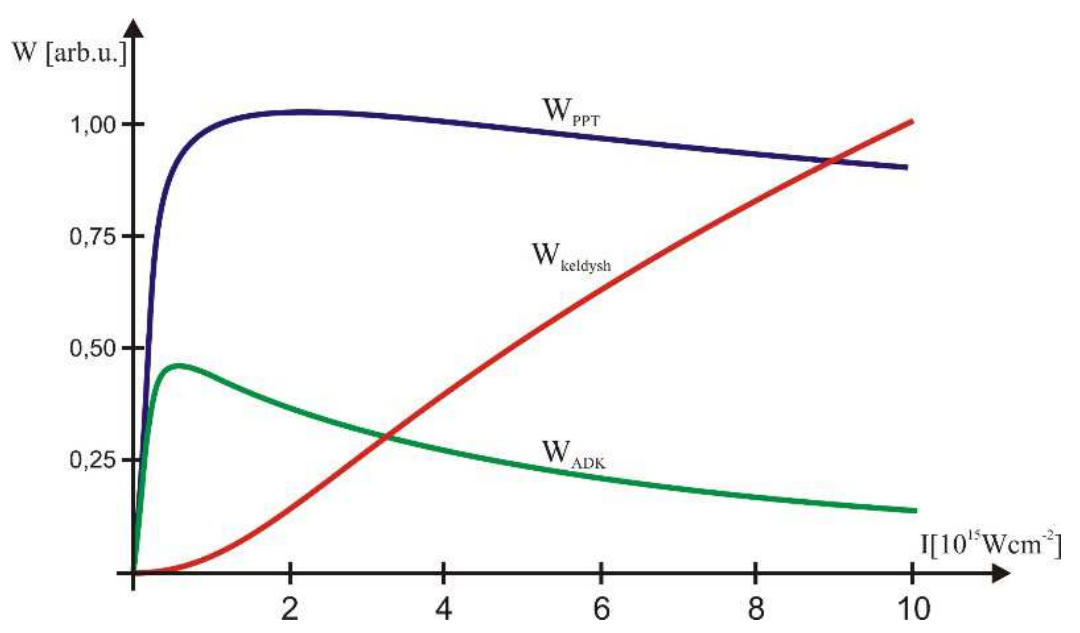

Figure 1. The ionization rates, $W_{\text {Keldysh }}, W_{P P T}, W_{A D K}$ vs laser field intensity, $I=10^{12}-10^{16} \mathbf{W e m}^{-2}$.

As can readily be seen, the field-free transition rate curves exhibit a significantly different behavior for the Keldysh, PPT and ADK theory. Keldysh curve almost linearly increases, while the other two reach some maximal value and then decrease. It is also obvious that PPT curve has the higher values of the transition rate compared to the ADK curve, for the same field intensity.

In order to perform a detailed analysis, the ponderomotive potential and the Stark shift were included in the equations for the transition rate (Eqs. 5, 6, 7). Here and in the following we used the notation: superscript " $U p$ " denotes an included ponderomotive potential, while "UpS" - ponderomotive potential and Stark shift. Fig. 2 demonstrates the dependence of the transition rates (with corrected ionization potential included) on the field intensity.
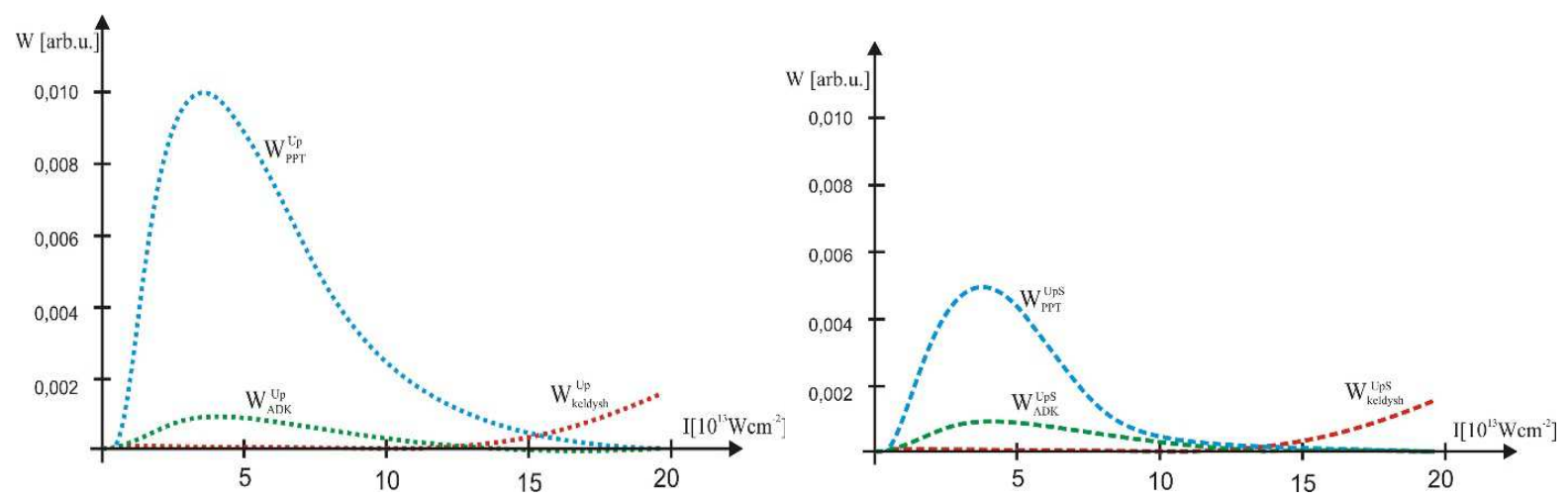

Figure 2. a) Left, the ionization rates, $W_{\text {Reldysh }}^{\mathbb{V}}, W_{F F}^{V F}, W_{A D K}^{V p}$ vs laser field intensity, $I=10^{12}-2 \times 10^{14} \mathrm{Wcm}^{-2}$; b) Right, the ionization rates, $W_{R E L I y s h}^{V P S}, W_{P F T}^{V p s}, W_{A D R}^{V p s}$ vs laser field intensity

$$
I=10^{13}-2 \times 10^{14} \text {. }
$$

All curves on Fig. 2 have the lower magnitude then on Fig. 1. The physical reason for this is the inclusion of the ponderomotive potential and the Stark effect which affect the ground state. In the intensity range under consideration, PPT curve is several orders of magnitude above the ADK, while Keldysh curve constantly increases in whole intensity range and so at the higher intensities have the higher values then PPT and ADK rates. It can be seen, by comparing left and right plot, that all curves have similar shape, but at different values of intensity. PPT and ADK curves have the maximum which is for the $W_{A D K}^{U_{p}}$, i.e. $W_{A D K}^{U p S}$ shifted to the lower field intensity when compared to $W_{P p T}^{U p}$, i.e. $W_{P F T}^{U p S}$. Also, the corresponding 
maximal values for $W_{A D K}^{U_{p} S}$ and $W_{P D T}^{U_{p} s}$ are reduced compared to $W_{A D K}^{U_{p}}$ and $W_{P F T}^{U_{p}}$ which can be expected because the ionization energy is shifted to higher values. Based on everything mentioned, it follows that inclusion of the additional parameters into formulas for the transition rates leads to the significant changes in the physical picture.

Next were plotted the curves for the Keldysh and the PPT transition rates, first without any corrections and then when all corrections are taken into account, respectively.
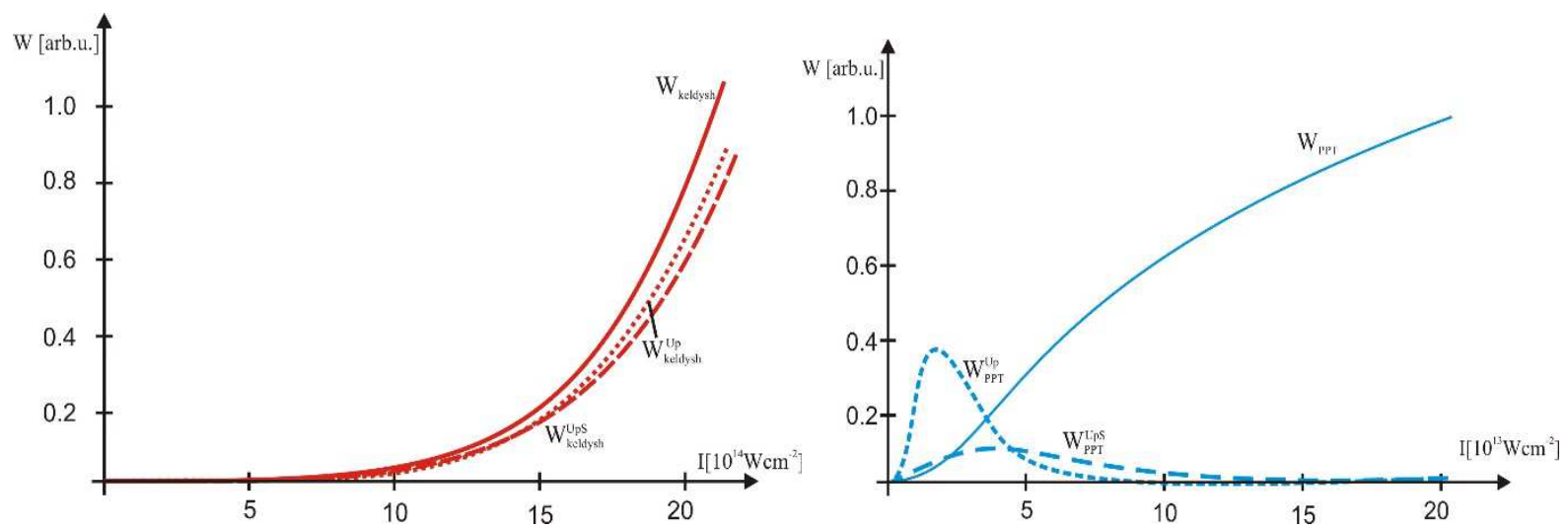

Figure 3: a) Left, the ionization rates $\boldsymbol{W}_{\text {Keldysh }}, \boldsymbol{w}_{\text {Kaldysh }}^{\text {Up }}, \boldsymbol{w}_{\text {Keldysh }}^{\text {UpS }}$ vs laser field intensity; b)Right, the ionization rates $W_{P P T} W_{P P T}^{U p}, W_{P P T}^{U p S}$ vs laser field intensity.

From Fig. 3, left plot, follows that all Keldysh curves, $w_{\text {Keldysh }}, w_{\text {Keldys }}^{U p}, w_{\text {Keldys }}^{U p s}$ have the same shape. Inclusion of the ponderomotive potential and the Stark shift decreases only the transition rate at the same field intensity. One would expect that the $\mathrm{K}$ transition rate curve is significantly influenced by the Stark shift, but the graph shows that the decreasing is very low, which isn't quite in accordance with theoretical prediction. Oppositely, right plot in Fig. 3 shows just the expected behavior of the PPT curve, as $w_{p p T}^{U p S}$ is significantly influenced by polarizability. Also, it is obvious that corrected $w_{p p T}^{U_{p}}, W_{p F T}^{U_{p S} S}$ differ strongly from the uncorrected $w_{P P T}$, and that with increasing laser field intensity the rates $w_{p F T}^{U p}, w_{p p T}^{U p S}$ approach zero. If the ponderomotive potential and the Stark shift of the energy of the ground state of an atom are both taken into account, the curves are shifted to the right, i.e. in the direction of lower field intensities.

Laser interaction with atoms depends on several parameters related with laser sources. One of these parameters is a spatial distribution of a laser beam. So, it is of interest how exactly the distribution influences the transition rates. In Fig. 4 are shown the compared results for Keldysh rates $w_{\text {Keldysh }}^{\text {eff }}$ and $W_{\text {Reldysh }}^{L_{\text {eff }}}$ (see Eq. 1 and Eq. 9), and PPT rates $w_{P P T}^{\text {eff }}$ and $W_{p p T}^{L_{1} \text { eff }}$ (see Eq. 2 and Eq. 10). Mark "L" in the superscript indicates the Lorentzian spatial distribution. This was accomplished for the corrections included. For the ADK theory the spatial dependence can be found in Petrović and MiLadinović (2014). The following figures were obtained. 

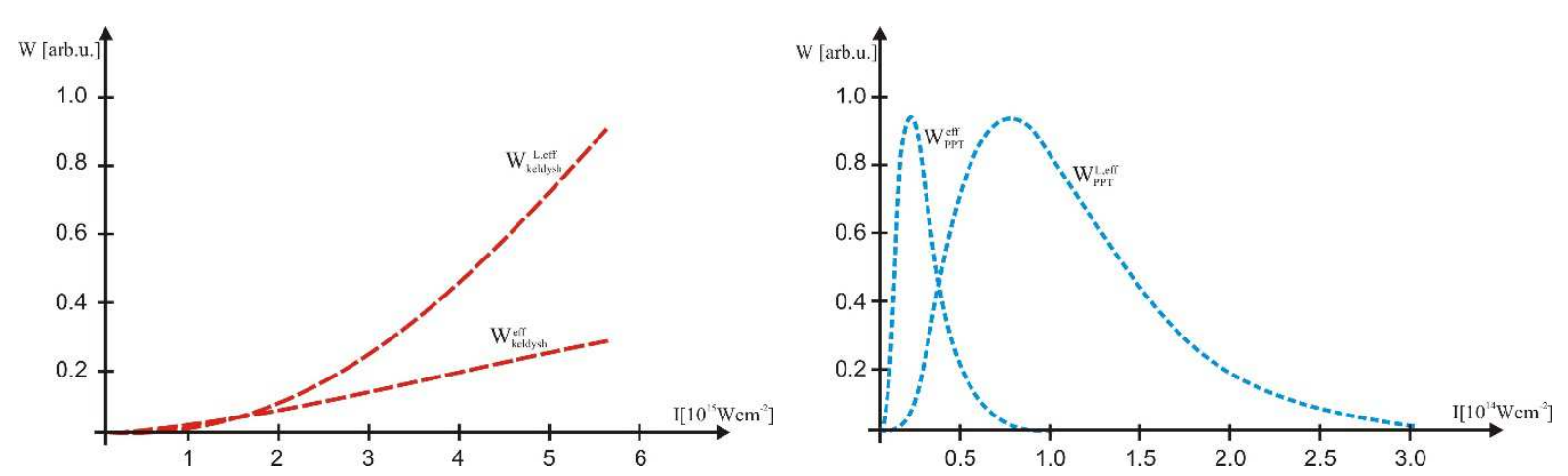

Figure 4. a) Left, $W_{\text {Reildysh }}^{\text {sff }}$ and $W_{\text {Reidysh }}^{2, \text { sff }}$ vs laser field intensity $I=10^{15}-6 \times 10^{15} \mathrm{Wem}^{-2}$;

b) Right, $W_{F F T}^{8 / f}$ and $W_{F F T}^{L, s f f}$ vs laser field intensity $I=10^{13}-3 \times 10^{14} \mathrm{Wcm}^{-2}$.

From Fig. 4 the influence of the spatial dependence is obvious. Both of Keldysh curves increase with increasing of the laser field intensity but this increase is much significant for $W_{\text {Reldish }}^{L, \text { aff }}$. As a consequence $W_{\text {Reldish }}^{L, \text { eff }}$ has higher values at $I>10^{15} \mathrm{Wcm}^{-2}$, compared to $W_{\text {Reldish }}^{\text {eff }}$ for the same field intensity.

The main conclusion that follows from the comparison of the curves $W_{p p T}^{\text {eff }}$ and $W_{p P T}^{L_{l} \otimes f f}$ is that the maximum for both transition rates is the same, but the curve $W_{p P T}^{L, E f f}$ reaches the maximum on the higher field intensity i.e. the maximum is shifted to the right. Both curves are asymmetric around the value of the field intensity on which $w_{F P T}^{\text {eff }}$ and $w_{P F T}^{L_{1, \theta f f}}$, respectively, have the maximum value, but this asymmetry is much more prominent for $W_{p F T}^{L_{1} \in f f}$. Both curves then approach zero with further increasing of the laser field intensity.

From aforementioned can be readily concluded that the transition rates are quite sensitive on the correction of the field free potential (potential without any corrections) and the laser beam shape, so the both effects must be taken into account.

Many useful data and interesting relations and dependences could be obtained from observed transition rates (on which field intensity the transition rate has the maximal value, how the Keldysh parameter influences on the transition rate, etc.). In order to provide a more detailed outlook on obtained results, an expert system logic was implemented on the aforementioned analyses, which resulted in a development of an expert system.

\section{EXPERT SYSTEM}

In this section we give a short review about the expert system developed for analysis of photoionization theories with respect on the issues described above. An expert system (sometimes referred to as knowledge-based system) is a computer software that emulates the decision-making ability of a human expert (MANDAL et al. 2013). Beside the specific expert systems building tools, such as I2, CLIPS, PROLOG, LISP, an expert system can also be developed by writing programs using certain programming languages, such as Fortran, Pascal, $\mathrm{C}++$ and Visual Basic (MuQueEM, 2014). The expert system was built on Visual Basic, while the accuracy of ES reasoning was partially checked through ESBT I2+. For testing of ES the available theoretical and experimental results were used.

We imitated the backward chaining logic where the system starts from a specific goal and attempts to satisfy the preconditions necessary for obtaining it (TILOTMA SHARMA et al. 2012). 
Interactions between the users and the system are supported through a friendly graphical user interface running under Windows environment. The system starts by initializing the first interface screen and prepare the "questions" for the user who must input some necessary, initial data such as laser wavelength $\lambda$, chemical element observed, range of laser field intensity $I$, the ion charge $\mathrm{Z}$, and Keldysh parameter, $\gamma$. All the listed data must be entered. Fig. 5 (left plot) shows the initial form of GUI.
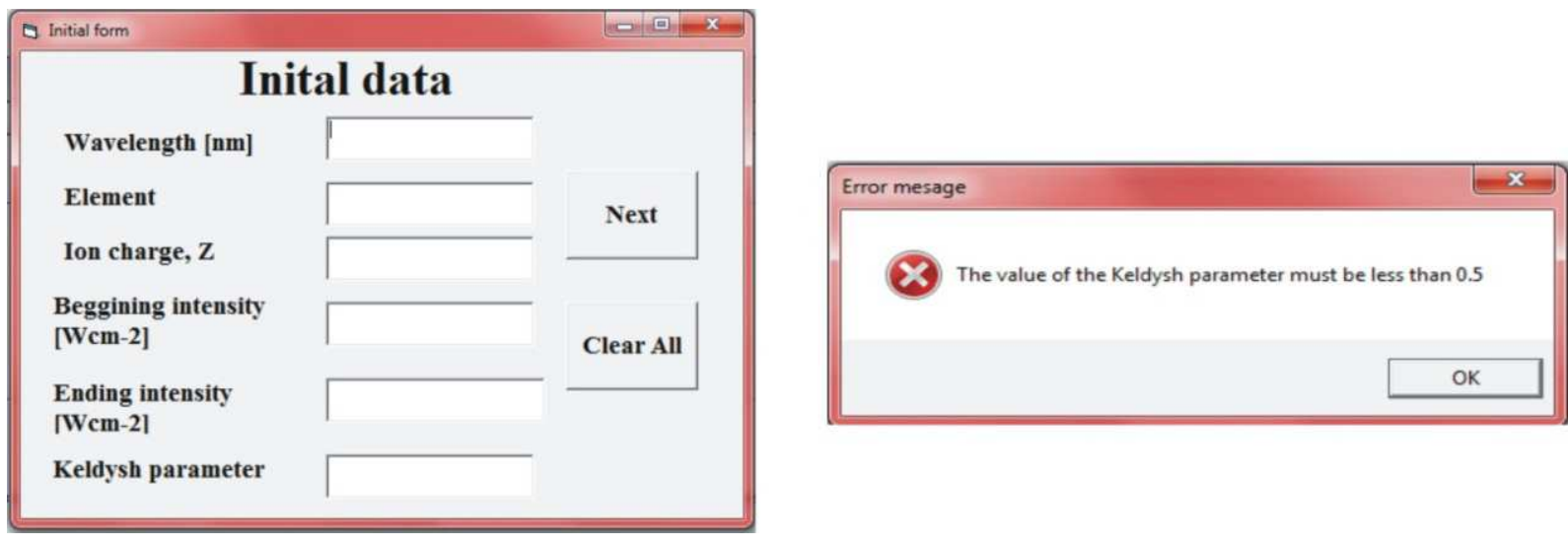

Figure 5: a) Left, the initial form, b) Right, an error message

After the user fills appropriate boxes, the ES then checks the data entered. If all data are valid, the ES continues to work. In an opposite case the "Error message" appears (right side on Fig. 5), so the user should re-enter a relevant data. For example, a valid value of the Keldysh parameter is less than 0.5 as the observed ionization process is that of tunneling ionization. The button "Clear all" deletes all entered data. The initial form completely corresponds to the structure and has the function of the initial rule. Initial rule is first activated rule in an ES. In characteristic "if then" logic, the corresponding initial rule has the following form:

RULE Initial

IF Initial conditions

AND Wavelength

AND Element

AND Ion charge

AND Intensity range

AND Keldysh parameter

THEN Initial data
ELSE Repeated input

AND FORGET Wavelength

AND FORGET Element

AND FORGET Ion charge

AND FORGET Intensity range

AND FORGET Keldysh parameter

AND CYCLE

The "IF" part of the initial rule contains premises bound with the logic operator "AND". In order to activate the rule all premises must be true (LUCAS and VAN DER GAAG, 1991).

It should be noted that we restricted ourselves only on above described issues. Otherwise, the ES can, based on the value of Keldysh parameter and intensity range conclude what ionization process is dominant and, based on that, perform appropriate analysis.

The "Next" button loads the form "Transition rate" with displayed various options, Fig. 6 (on left side): 

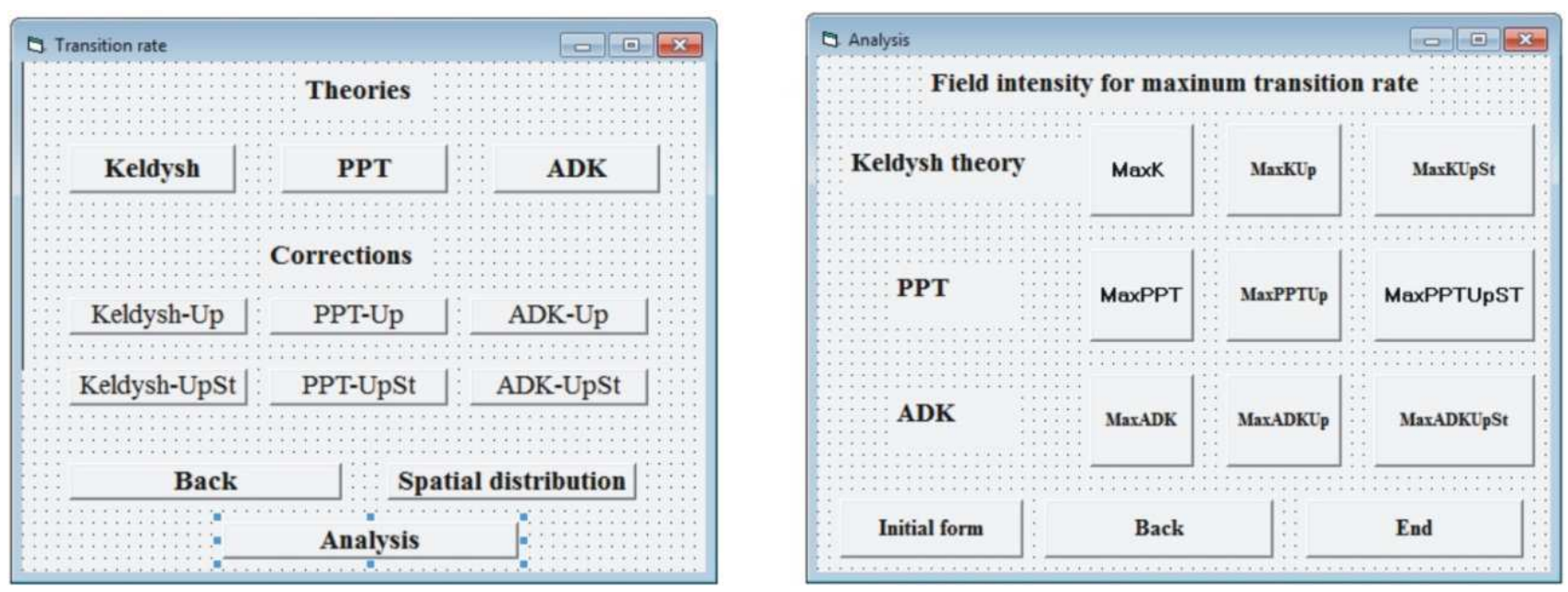

Figure 6. The form "Transition rate"

Active buttons enable a calculation of transition rates by different theories, without or with corrections (see Section 2). Obtained results are then written in the table "Transition rates" that has a following structure:

Table 1. The structure of table for writing data.

\begin{tabular}{|c|c|c|c|c|c|c|c|c|c|}
\hline \multirow{2}{*}{$\begin{array}{l}\text { Intensity } \\
{\left[\mathrm{Wcm}^{-2}\right]}\end{array}$} & \multicolumn{3}{|c|}{ Transition rates without correction } & \multicolumn{6}{|c|}{ Transition rates with correction } \\
\hline & Keldysh & PPT & ADK & KeldyshUp & PPTUp & ADKUp & KeldyshUpSt & PPTUpSt & ADKUpSt \\
\hline
\end{tabular}

Additionally, the form contains the button "Analysis" that calls a form Analysis shown on right side of Fig. 6, that offers the procedures necessary to determine on which laser field intensities the maximal values of the transition rates appear. "Back" option enables a return to the previous form; also the user can select some other options. Each button on the form activates a subroutine for the analysis of that particular transition rate. Obtained results are then written in appropriate columns of the "Transition rates" table.

If the case when all of the transition rates are calculated, the following, completely filled table, is obtained:

Table 2. A filled table, with fields that are given appropriate values during the work of the ES.

\begin{tabular}{|c|c|c|c|c|c|c|c|c|c|}
\hline \multirow{2}{*}{$\begin{array}{c}\text { Intensity } \\
{[\mathrm{Wcm}-2]}\end{array}$} & \multicolumn{3}{|c|}{ Transition rates without correction } & \multicolumn{6}{|c|}{ Transition rates with corrections } \\
\hline & Keldysh & PPT & ADK & KeldyshUp & PPTUp & ADKUp & KeldyshUpSt & PPTUpST & ADKUpST \\
\hline$\ldots$ & $\ldots$ & $\ldots$ & $\ldots$ & $\ldots$ & $\ldots$ & $\ldots$ & $\ldots$ & $\ldots$ & $\ldots$ \\
\hline $5 \mathrm{E}+13$ & $5,20478 \mathrm{E}-09$ & $9,26985 \mathrm{E}-09$ & $2,62715 \mathrm{E}-10$ & $5,09141 \mathrm{E}-09$ & $1,49514 \mathrm{E}-11$ & $4,77238 \mathrm{E}-13$ & $5,08808 \mathrm{E}-09$ & $1,17562 \mathrm{E}-11$ & $3,76608 \mathrm{E}-13$ \\
\hline $6 \mathrm{E}+13$ & $2,46988 \mathrm{E}-08$ & $5,81753 \mathrm{E}-08$ & $1,66435 \mathrm{E}-09$ & $2,41284 \mathrm{E}-08$ & $4,82734 \mathrm{E}-11$ & $1,59615 \mathrm{E}-12$ & $2,41122 \mathrm{E}-08$ & $3,69449 \mathrm{E}-11$ & $1,22683 \mathrm{E}-12$ \\
\hline $7 \mathrm{E}+13$ & $8,3105 \mathrm{E}-08$ & $2,41731 \mathrm{E}-07$ & $6,9595 \mathrm{E}-09$ & $8,10962 \mathrm{E}-08$ & $1,07449 \mathrm{E}-10$ & $3,66649 \mathrm{E}-12$ & $8,10409 \mathrm{E}-08$ & $8,01369 \mathrm{E}-11$ & $2,74805 \mathrm{E}-12$ \\
\hline $8 \mathrm{E}+13$ & $2,21513 \mathrm{E}-07$ & $7,60255 \mathrm{E}-07$ & $2,19814 \mathrm{E}-08$ & $2,1596 \mathrm{E}-07$ & $1,86738 \mathrm{E}-10$ & $6,55836 \mathrm{E}-12$ & $2,15812 \mathrm{E}-07$ & $1,35844 \mathrm{E}-10$ & $4,79742 \mathrm{E}-12$ \\
\hline $9 \mathrm{E}+13$ & 4,99861E-07 & $1,96052 \mathrm{E}-06$ & $5,68464 \mathrm{E}-08$ & $4,86957 \mathrm{E}-07$ & $2,72767 \mathrm{E}-10$ & 9,83947E-12 & $7,02924 \mathrm{E}-12$ & $1,93679 \mathrm{E}-10$ & $4,86623 \mathrm{E}-07$ \\
\hline $1 \mathrm{E}+14$ & $9,96505 \mathrm{E}-07$ & $4,36309 \mathrm{E}-06$ & $1,26742 \mathrm{E}-07$ & $9,70153 \mathrm{E}-07$ & $3,50845 \mathrm{E}-10$ & $1,29777 \mathrm{E}-11$ & $9,69491 \mathrm{E}-07$ & $2,43295 \mathrm{E}-10$ & $9,05904 \mathrm{E}-12$ \\
\hline $2 E+14$ & $4,30719 \mathrm{E}-05$ & 0,000321848 & $9,3063 \mathrm{E}-06$ & $4,18143 \mathrm{E}-05$ & $2,0159 \mathrm{E}-10$ & $9,06025 \mathrm{E}-12$ & $4,17897 \mathrm{E}-05$ & $1,11886 \mathrm{E}-10$ & $5,09089 \mathrm{E}-12$ \\
\hline $3 E+14$ & 0,000234167 & 0,00211086 & $6,00454 \mathrm{E}-05$ & 0,000227293 & $1,74199 \mathrm{E}-11$ & $9,03807 \mathrm{E}-13$ & 0,000227183 & $7,86587 \mathrm{E}-12$ & $4,12973 \mathrm{E}-13$ \\
\hline $4 E+14$ & 0,000649923 & 0,00640299 & 0,000178871 & 0,000631232 & $8,72133 \mathrm{E}-13$ & $5,04752 \mathrm{E}-14$ & 0,00063098 & $3,21068 \mathrm{E}-13$ & $1,88154 \mathrm{E}-14$ \\
\hline $5 E+14$ & 0,00131317 & 0,0135639 & 0,000372118 & 0,0012765 & $3,43905 \mathrm{E}-14$ & $2,17185 \mathrm{E}-15$ & 0,00127607 & $1,03428 \mathrm{E}-14$ & $6,61385 \mathrm{E}-16$ \\
\hline & & & & & & & & & \\
\hline
\end{tabular}


There is not the only given return from the ES. The user also can decide in which moment he wants an analysis to finish. The ending rule given below illustrates that.

RULE End

IF Initial data

AND Transition rates
OR Analysis

THEN Analysis completed

The rule will be activated if all of needed premises linked through the logic "AND" operator are satisfied and also if at least one premise linked through the logic "OR" operator is satisfied.

\section{CONCLUSION}

The ionization rates of alkali atoms by three theories, Keldysh, PPT and ADK for the case of non-relativistic linearly polarized laser field were studied. It was shown that the ponderomotive potential and the Stark shift influence the transition rates, even in the case of alkali atoms with relatively small polarizability. It was also shown that an expert system can be used for an analysis of the transition rate. The described system can be improved in several ways and this will be further pursued in forthcoming papers.

\section{Acknowledgements}

We are grateful to the Serbian Ministry of Education and Science for financial support through Project 171020.

\section{References}

[1] Ammosov, V.M., Delone, N.B., Krainov, V.P., Tunnel ionization of complex atoms and atomic ions by an alternating electromagnetic field, Soviet Physics JETP 64 (6) (1986) 11911194.

[2] BAUER, D. Theory of intense laser-matter interaction, Max-Planck Inst., Heidelberg, Germany, (2006) 58, http://www.physik.uni-rostock.de/fileadmin/Physik/Bauer/tilmi.pdf

[3] Delone, N.B., Krainov, V.P., Multiphoton Processes in Atoms, 2nd edition, Springer (2000).

[4] Delone, N.B., KRAINOV, V.P., Tunneling and barrier-suppression ionization of atoms and ions in a laser radiation field, Physics Uspekhi 41 (5) (1998) 469-485.

[5] KeLDYSH, L.V., Ionization in the field of a strong electromagnetic wave, Soviet Physics JETP 20 (5) (1964) 1945-1957.

[6] Landau, L.D., LIFshitZ, E.M., Quantum Mechanics: Non-Relativistic Theory, $3^{\text {rd }}$ ed. Pergamon, Oxford, (1991) 295-298., https://issuu.com/sonocontentodite/docs/landau-l.d.--lifschitz-e.m.--vol.-1---mechanics-3

[7] Lin, S.H., Villaeys, A.A., Fujimura Y., Advances in Multi-photon Processes and Spectroscopy 16, Singapore: World Scientific Publishing Co. Pte. Ltd, (2004) 249 pp, 
[8] LuCas, P.J.F., VAn Der GaAg, L.C., Principles of Expert Systems, Centre for Mathematics and Computer Science, Amsterdam, Addison-Wesley (1991).

[9] Mandal S., Chatterjee S., Neogi B., Diagnosis and troubleshooting of computer faults based on expert system and artificial intelligence, International Journal of Pure and Applied Mathematics 83 (5) (2013) 717-729,

[10] MuQueEM, S., Expert system application in library, Knowledge Librarian - An International Peer Reviewed Bilingual E-Journal of Library and Information Science, 1 (2) (2014) 168-175.

[11] Perelomov, A.M., Popov, V.S., Terent'Ev, M.V., Ionization of atoms in an alternating electric field, Soviet Physics JETP, 23 (5) (1966) 924-934.

[12] Petrović, V.M., Miladinović, T.B., Influence of the spatial and temporal distribution of an incident laser beam profile on the energy distribution of ionized photoelectrons, JETP 119 (4) (2014) 651-656.

[13] Ristić, V.M., Miladinović, T.B., Radulović, M.M., Calculating ionization transition rate for circularly polarized fields, including non-zero initial momentum, Acta Phys. Pol. A 116 (4) (2009) 504-506.

[14] ShCHATSININ IHAR, Free clusters and free molecules in strong, shaped laser fields, Universität Berlin, PhD Thesis (2009) 51-71.

[15] SCHWERDTFEgER, P., Table of experimental and calculated static dipole polarizabilities for the electronic ground states of the neutral elements (in atomic units) (2014), http://ctcp.massey.ac.nz/Tablepol2014.pdf

[16] Shealy, D.L., Hoffnagle, J.A., Laser beam shaping profiles and propagation. Applied Optics 45 (21) (2006) 5118-5131 DOI: 10.1364/AO.45.005118

[17] Tilotma Sharma, Navneet Tiwari, Deepali Kelkar, Study of difference between forward and backward reasoning, International Journal of Emerging Technology and Advanced Engineering 2 (10) (2012) 271-273.

[18] Volkova, E.A., Gridchin, V.V., Popov, A.M., Tikhonova, O.V., Tunneling ionization of a hydrogen atom in short and ultrashort laser pulses, JETP 102 (1) (2006) 40-52.

[19] Volkova, E.A., Popov, A.M., Tikhonova, O.V., Ionization and stabilization of atoms in highintensity, low-frequency laser field, JETP 113 (3) (2011) 394-406.

[20] ZHANG, L., Intensity spatial profile analysis of a Gaussian laser beam at its waist using an optical fiber system, Chinese Physics Letters 27 (5) (2010) 054207-3 